\title{
MedienPädagogik
}

Zeitschrift für Theorie und Praxis der Medienbildung

\section{Medienpädagogische Kompetenz als Grundqualifikation in pädagogischen Berufen}

\author{
Dieter Spanhel
}

\section{Ausgangsthese}

Professionelles pädagogisches Handeln findet immer in sozialen Systemen statt, die sich auf der Grundlage von Kommunikationsprozessen konstituieren. Als pädagogische Handlungssysteme haben sie eine bestimmte Organisationsform gemeinsam, die sie von anderen Handlungssystemen unterscheidet. Die These von der medienpädagogischen Kompetenz als Grundqualifikation in pädagogischen Berufen geht davon aus, daß sich durch die Medienentwicklungen in der modernen Medien-, Informations- oder Wissensgesellschaft sowohl Organisationsform als auch Kommunikationsprozesse pädagogischer Handlungssysteme gewandelt haben.

Durch das Eindringen der Medien in alle Bereiche des menschlichen Alltagslebens ist das berufliche pädagogische Handeln auf zweierlei Weise betroffen: Zum einen indirekt dadurch, daß sich durch die mediengeprägte Lebenswelt die Voraussetzungen und Ziele, die Bedingungen, die Aufgaben und Inhalte des professionellen pädagogischen Handelns ändern. Zum anderen direkt dadurch, daß Medien auch in alle Erziehungsinstitutionen und Felder professionellen pädagogischen Handelns Eingang gefunden haben und die Möglichkeiten, Formen, Methoden und Strukturen dieses kommunikativen Handelns beeinflussen. Daraus ergibt sich zwingend die Notwendigkeit einer medienpädagogische Grundqualifikation für pädagogische Berufe.

Die Überprüfung der These erfordert einen theoretischen Bezugsrahmen zur genauen Beschreibung der Organisation und Struktur von beruflichen pädagogischen Handlungssituationen. Ich verwende dafür ein Strukturmodell sozialer Systeme, das A. Hermanns (1995, S. 68) in Anlehnung an N. Luhmann (1985) zur Beschreibung von Erziehungssystemen entwickelt hat. Demnach läßt sich jedes pädagogische Handlungssystem durch vier Grenzparameter kennzeichnen, über die es mit seiner Umwelt, d.h. mit der Gesellschaft und anderen sozialen Systemen in Kontakt steht. Im „Inneren“ funktioniert ein solches System auf der Grundlage pädagogischer Kommunikation, die in ihrem Ablauf durch drei Strukturparameter bestimmt wird. Meine These ist nun die, daß in der heutigen Medienwelt und durch eine praktisch unvermeidliche Mediennutzung sich sowohl die Grenz- als auch die Struk- 
turparameter wandeln. Beide Dimensionen sind jedoch nicht unabhängig voneinander: Die Qualität der Kontakte nach außen (Grenzparameter) beeinflußt die innere Struktur (Strukturparameter) des Handlungssystems und umgekehrt. Dies möchte ich im folgenden genauer aufzeigen.

\section{Zur Begründung der Notwendigkeit einer medienpädagogischen Grundquali- fikation}

Ich untersuche zunächst die Bedeutung der Medien für die Außenkontakte pädagogischer Handlungssysteme. Der erste Grenzparameter betrifft die veränderte gesellschaftliche Funktion professionellen pädagogischen Handelns in einer von den elektronischen Medien geprägten Welt.

Insbesondere die Digitalisierung der Medien und die Entwicklung von Multimedia beschleunigen den wissenschaftlich-technischen Fortschritt und daraus folgende tiefgreifende wirtschaftliche, gesellschaftliche und soziale Wandlungen. Sie werden meist mit den Merkmalen Beschleunigung, Vernetzung, Globalisierung, Individualisierung und Pluralität, Entstehung virtueller Welten sowie Verfügbarkeit, Beliebigkeit und rasche Veralterung des Wissens beschrieben. Schon heute resultieren daraus während des gesamten Lebenslaufs neue Lernzwänge und Lerninhalte, Entwicklungs- und Sozialisationsprobleme sowie Notwendigkeiten zum Umlernen und Weiterlernen (insbesondere z.B. im beruflichen Bereich), aber auch psychische Belastungen und Verhaltensstörungen (z.B. Angst, Aggressionen und Gewalt als Folge von Medieneinwirkungen).

Immer mehr zeichnet sich in der modernen Wissensgesellschaft zudem die Notwendigkeit eines lebenslangen Lernens für alle Menschen ab, um die schwierigen Probleme in einer nicht vorhersehbaren Zukunft bewältigen zu können. Zusätzlich zur Tradierung eines systematisierten Grundbestands bewährten Wissens werden daher künftig die Formen des Lernens, die Qualität der Lernmethoden, das Lernen lernen, Weiterlernen, Umlernen und die Fähigkeit zum Umgang mit dem Nichtwissen immer wichtiger. Es geht dabei um den Aufbau variabler Lerninstrumente bei den Lernenden, d.h. um operatives Wissen, um breites und abstraktes Orientierungswissen und um die Fähigkeit zum flexiblen Einsatz dieser Wissensstrukturen auf der Grundlage von vernetztem Denken, von Phantasie und Kreativität (vgl. Spanhel 1994).

Die besondere gesellschaftliche Funktion des professionellen pädagogischen Handelns muß in diesem Zusammenhang dann beschrieben werden als die Bereitstellung spezifischer, auf den ganzen Lebenslauf bezogener Lernhilfen (vgl. H. Giesecke 1996). Aber nicht nur als Lernhilfen zur selbständigen Bewältigung dieser Lern- und Entwicklungsaufgaben, Alltags- und Lebensprobleme in jeder Altersphase, sondern als spezifische fachliche Hilfen zum Lernen des Lernens, zur Erhaltung und Motivierung einer lebenslangen Lernbereitschaft, zum Weiter- und Umlernen, zur Weiterentwicklung der Lerninstrumente, um auf diese Weise einen Beitrag zu leisten, diese gesellschaftlichen 
Entwicklungen human zu gestalten, die Schlüsselprobleme unserer Gesellschaft zu lösen und neue Herausforderungen in einer nicht vorhersehbaren Zukunft bewältigen zu können (vgl. Forum Info 2000, 1998, S. 7ff.).

Wir könnten mit den Worten von A. Treml von Hilfe für ein „Lernen der Gesellschaft“ sprechen, das er folgendermaßen kennzeichnet: „Heutzutage muß ein Individuum immer weniger feste kulturelle Bildungsgüter lernen, sondern vor allem lernen, mit der gesellschaftlich erzeugten Selektivität und Kontingenz umzugehen. Es kann jetzt nicht mehr darum gehen, Wissen unter den Bedingungen der Gewißheit und Sicherheit anzuhäufen, sondern vielmehr zu lernen, in der Zeitdimension mit Gewißheit und Ungewißheit, in der Sachdimension mit Wissen und Nichtwissen und in der Sozialdimension mit Sicherheit und Unsicherheit umzugehen. Es wird also vermutlich zunehmend um ein abstraktes Lernen des Lernens gehen - ein Lernen, das weniger an Inhalten, als vielmehr durch Kontexte gelernt wird und Veränderungen grundlegender (insbesondere normativer) Erwartungen erzwingt“ (Treml 1995, S. 101; Hervorh. durch den Autor).

Der zweite Grenzparameter beschreibt die Kontakte pädagogischer Handlungssysteme zu den gesellschaftlichen Systemen, von denen sie bestimmte Leistungen empfangen. Die Inputleistung besteht in den Voraussetzungen, die andere gesellschaftliche Systeme für pädagogische Handlungssysteme erbringen. Dazu gehören z.B. nicht nur die Leistungen des politischen Systems für den Betrieb der Erziehungs- und Bildungseinrichtungen und die fachliche Aus- und Fortbildung der Berufspädagogen, sondern immer mehr das Mediensystem und die explodierenden Medienangebote in der Alltagswelt der Menschen:

- Medien nehmen heute einen immer größeren Teil der Lebenszeit der Menschen jeden Alters in Anspruch und wirken auf Zeitplanung und Lebensrhythmus ein.

- Medien bringen eigene Wirklichkeiten hervor, zu denen der Mensch Stellung beziehen muß und führen zur Erweiterung der Erfahrungen, Weltsichten, Lebensstile und Sozialkontakte.

- Medien bieten eine verwirrende Vielfalt von Sinn- und Lebensperspektiven, Normen und Werten sowie widersprüchliche Handlungsorientierungen.

- Medien eröffnen neue Chancen und Formen des Kontakts und der Auseinandersetzung des Menschen mit seiner Welt, der Weltaneignung und des Aufbaus eines eigenen Weltbilds.

- Medien dienen der Erweiterung des persönlichen Erlebens, des Ausdrucks und der Selbstdarstellung sowie der Entwicklung und Sicherung der eigenen Identität.

Professionelle Pädagogen müssen also erkennen und möglichst genau bestimmen können, auf welche Weise und in welchem Umfang die folgenden grundlegenden Bedingungen der pädagogischen Kommunikationsprozesse in ihren Berufsfeldern von Medien geprägt werden: 
- die Lebenssituation und das soziale Umfeld ihrer Klienten,

- deren Bedürfnisse und Emotionen, ihr Wissen und ihre Erfahrungen,

- ihre Urteilsfähigkeit und ihre Wertorientierungen und insbesondere

- die von ihnen bereits aufgebauten kommunikativen Fähigkeiten.

Sie müssen wissen, wie sie an diese Voraussetzungen ihrer Klienten anknüpfen und sie für eine fruchtbare Gestaltung der angestrebten Lern- und Bildungsprozesse nutzen können.

Damit kommt der dritte Grenzparameter in den Blick, die Outputleistung pädagogischer Handlungssysteme. Hier geht es um die pädagogischen Ziele, die für eine Informations- und Wissensgesellschaft kennzeichnend sind. Damit werden die Leistungen beschrieben, die Berufspädagogen zur Integration ihrer Klienten in befriedigende Sozialbeziehungen, in das Berufs-, Wirtschafts- und Hochschulsystem, in das politische und das Mediensystem und zu ihrer verantwortlichen Teilhabe am gesellschaftlichen und kulturellen Leben und zu dessen Weiterentwicklung erbringen sollen. Diese Ziele liegen auf zwei Ebenen: Zum einen müssen heute die Menschen jeden Alters zu einem kritischen, sachgerechten und eigenverantwortlichen Umgang mit den Medien befähigt werden. Zum anderen können und müssen die alten und neuen Medien als Werkzeuge zur Verbesserung der Lern-, Erziehungs- und Bildungsprozesse, in ihren Möglichkeiten zur Lebensbereicherung, Selbstverwirklichung und Unterhaltung, zur Erleichterung von Aufgaben- und zur Problembewältigung sowie zur Vermittlung von Schlüsselqualifikationen, zur Aus- und Weiterbildung im Beruf eingesetzt werden (vgl. Tulodziecki/ Blömeke 1997, S. 34f. und S. 155f.).

Um die ganze Bandbreite der Medien zur Ausgestaltung und Bereicherung der Kommunikationsprozesse in der Gesellschaft adäquat einsetzen zu können, sind eine Vielzahl von Fähigkeiten erforderlich. Grundlegend sind die kommunikativen Fähigkeiten, auf denen jedes soziale System aufbaut. Dazu gehören die Fähigkeiten zur Verständigung über Inhalte, zur Herstellung und Aufrechterhaltung sozialer Beziehungen und zum Aufbau und zur Erhaltung der Identität mittels unterschiedlicher Zeichensysteme und Medien (vgl. Boeckmann 1994). Hinsichtlich der einzelnen Medien mit ihren spezifischen Leistungen müssen die Menschen darüber hinaus Fähigkeiten aneignen, die unter dem Terminus Medienkompetenz zusammengefaßt werden können. (Obwohl der Begriff der Medienkompetenz sehr unterschiedlich und auch kritisch gesehen wird, sollte er dennoch als Zusammenhang stiftende Vokabel und gemeinsame Zielformel beibehalten werden; vgl. z.B. von Rein, 1996; Moser, 1999.

Nach Auffassung von Moser verweist der Begriff Medienkompetenz darauf, daß es „eine Grundproblematik über die einzelnen Medien hinweg (gibt), mit denen Menschen kompetent umgehen müssen, wenn sie in der zukünftigen Informationsgesellschaft bestehen wollen“. Es gehe dabei „um breite Basisqualifikationen, die für das alltägliche Leben des aktiven Bürgers in Zukunft unentbehrlich sein werden“. Der Begriff sei von den Lernenden 
her gedacht, er argumentiere nicht defizitär, sondern mit einem „Können“: „Unter konstruktivistischen Vorzeichen wäre deshalb gerade die Medienkompetenz als ein Bündel von Fähigkeiten zu entwerfen, das die Lernenden im Umgang mit Medien selbst zu entwickeln haben - im Rahmen von Lernsituationen und Arrangements, für die gilt: Denken, Lernen und Wissenserwerb, das bedeutet nicht, Vorgegebenes abzubilden, sondern Eigenes zu gestalten (Reinmann-Rothmeier/Mandl 1997)“ (Moser 1999, S. 215f.). Mosers Begriff von Medienkompetenz umfaßt Fähigkeiten auf folgenden vier Ebenen:

1) Technisch als Notwendigkeit, Medien richtig zu handhaben und die mit ihnen verbundenen Gestaltungsmöglichkeiten zu beherrschen.

2) Kulturell als Vertrautsein mit den jeweiligen Codes der Medien sowie mit ihren ästhetischen und gesellschaftlichen Ausdrucksformen.

3) Sozial als Fähigkeit, auf die mit den Medien verbundenen Kommunikationsangebote und -Zumutungen sinnvoll eingehen zu können.

4) Reflexiv als kritische Vergewisserung der Funktion der Medien in der Gesellschaft und als Medienkritik (vgl. Moser 1999, S. 216f.).

Dieses Bündel von Fähigkeiten ließe sich auch umschreiben als Wahrnehmungs- und Verarbeitungskompetenz, als Beurteilungs- und Selektionskompetenz, als kritische Nutzungs- und kreative Handlungskompetenz. Mit Bezug auf die neuesten Entwicklungen dürfte eine multimediale Kompetenz zur optimalen Nutzung hypermedialer Lernsysteme in Zukunft zunehmende Bedeutung gewinnen (vgl. Aufenanger 1996) und darüber hinaus die moralische Kompetenz, für die Folgen des Medienhandelns die Verantwortung zu übernehmen (vgl. dazu auch Tulodziecki 1995, S. 23ff.; Aufenanger 1992).

Der vierte Grenzparameter wird als Interpenetration bezeichnet. Damit ist die Schnittstelle zwischen dem professionellen pädagogischen Handlungssystem und dem psychischen System der einzelnen Klienten angesprochen. Es geht dabei um die Frage, wie die Klienten mit ihren kognitiven, emotionalen, affektiven und psychomotorischen Schemata an die pädagogischen Kommunikationsstrukturen anknüpfen können bzw. wie diese die psychischen Dispositionen beeinflussen und welche Lernprozesse dadurch ausgelöst und gefördert werden.

Dazu muß zunächst klar sein, daß Lernen aus systemtheoretischer Sicht immer ein eigenaktiver und konstruktiver Prozeß ist. Nach dieser Auffassung werden beim Lernen keine fertigen Inhalte übergeben und aufgenommen. Vielmehr konstruieren die Lernenden mit Hilfe der bereits vorhandenen und gerade verfügbaren Schemata neue Wissensstrukturen. Aus der Vielfalt der Wahrnehmungen in der Auseinandersetzung mit der Umwelt abstrahieren sie neue Muster, die zu Transformationen der vorhandenen Denk-, Gefühls-, Wertungs- und Handlungsmuster führen. Sie sind die Instrumente (in Form von Bewegungs- oder Handlungsmustern, Begriffen oder Theorien), mit denen gelernt werden kann. Die Gesamtheit der inneren Strukturen bildet also den „Lernapparat“ des Menschen (in Anlehnung an K. Lorenz, der von 
„Weltbildapparat“ spricht; vgl. dazu Lassahn 1977, S. 173ff.), der in der Kindheit und Jugend aufgebaut und das ganze Leben hindurch weiter entwickelt werden muß.

Die Aneignung konkreter Inhalte führt immer zur Transformation der vorhandenen Schemata. Dabei kommt es eben nicht nur auf die Aneignung neuen Wissens an, sondern entscheidend ist die Qualität der Lernprozesse und die Fähigkeit, das Lernen zu lernen, d.h. das bewußte und flexible Zurechtmachen, die Neukoordination, Ausdifferenzierung und damit Weiterentwicklung dieses Lernapparats in der Auseinandersetzung mit den Anforderungen der medialen Umwelt. Dieser selbstgesteuerte Entwicklungsprozeß auf der Basis sozialer Kommunikation ist problemorientiert und kontextabhängig. Lernen kann daher nicht direkt gelenkt, sondern nur durch die Gestaltung von Lern- und Erfahrungsräumen angeregt, ausgelöst, motiviert und begleitet werden (vgl. Spanhel 1994).

Gerade dafür bieten die Vielfalt und Verfügbarkeit der Medien, ihre Kombinierbarkeit mittels Computer und insbesondere Multimedia und Internet eine Fülle neuer Möglichkeiten, spezifische Lernumgebungen so zu gestalten, daß die Kommunikationsprozesse erheblich differenzierter und komplexer werden. Im Umgang mit den schier unbegrenzten Möglichkeiten zur Information und Kommunikation mittels Internet und Email werden Selbständigkeit und Eigenverantwortung beim Lernen und Problemlösen gefördert. Die Möglichkeiten zur Aufnahme und Speicherung, Verarbeitung und Aufbereitung, Darstellung und Weitergabe von Informationen mit Hilfe von MultimediaComputer erlauben es Lehrenden und Lernenden, Erwachsenen und Kindern, ihre Lernarrangements eigentätig und nach ihren individuellen Vorstellungen zu gestalten.

Dabei darf jedoch nicht vergessen werden, daß die neuen Medien den Lernenden die eigentliche Lernarbeit nicht abnehmen können und daß sie die Gefahr einer Vernachlässigung der sozialen und emotionalen Komponente des Lernens und der Manipulation der Lernenden mit sich bringen. Eine medienpädagogische Qualifikation verlangt damit ein mediendidaktisches und methodisches Grundwissen, um die Lernpotentiale der einzelnen Medien erkennen, richtig einschätzen und zur besseren Bewältigung spezifischer Lern-, Erziehungs- oder Bildungsaufgaben oder Problemlösungen einsetzen zu können.

\section{Veränderungen in der Kommunikationsstruktur pädagogischer Handlungs- felder}

Pädagogische Arbeitsfelder als soziale Systeme beruhen nach N. Luhmann ausschließlich auf Kommunikationsprozessen. Sie lassen sich in Anlehnung an den gewählten Bezugsrahmen (vgl. Hermanns 1995) mit Hilfe der sog. Strukturparameter hinsichtlich ihrer Sachstruktur, Sozialstruktur und Zeitstruktur genauer beschreiben. Sie bestimmen die Art und Weise, wie in päd- 
agogischen Handlungssystemen Kommunikationen aneinander angeschlossen werden können („Anschlußrationalität“, Hermanns 1995, S. 67).

Ich möchte nun im zweiten Schritt untersuchen, wie sich diese Strukturparameter pädagogischer Handlungsfelder in einer mediengeprägten Alltagswelt bzw. durch Medieneinsatz verändern und welche neuen beruflichen Anforderungen sich daraus ergeben. Dabei beziehe ich mich auf die Merkmale pädagogischer Professionalität und die Dimensionen eines pädagogischen Handlungsrepertoires, wie sie von K.O. Bauer (1998) beschrieben worden sind.

3.1 Veränderung des professionellen pädagogischen Handelns im Zusammenhang mit der Veränderung der Sachstruktur

Die Sachstruktur pädagogischer Handlungsfelder und damit die Inhalte pädagogischer Kommunikationsprozesse werden heute in vielfacher Weise durch Medien mitbestimmt:

- Durch unterschiedliche mediale Präsentation bzw. Repräsentation der Kommunikations-Inhalte. Jedes Medium beruht auf einem bestimmten Zeichensystem oder einer Kombination aus Zeichen und prägt in spezifischer Weise die Qualität der Kommunikationsprozesse. Es spricht daher auf unterschiedliche Weise die Wahrnehmungsfähigkeit, die Gefühle und das Unbewußte (Bilder und Töne), das Vorstellungsvermögen (Computersimulationen) oder das begrifflich-abstrakte Denken (Sprache) an. Medien

- konstituieren bzw. rekonstruieren Wirklichkeit in vorstellungsmäßigen oder begrifflichen, konkreten oder abstrakten Formen und können den Lernenden damit den Zugang zu schwierigen Sachverhalten und die Ausdifferenzierung ihres Weltbilds erleichtern;

- stiften und vermitteln Sinn und liefern so den Klienten eine Fülle an möglichen Sinnorientierungen, an die sie ihre individuellen Vorstellungen und Wünsche, Sehnsüchte und Hoffnungen anschließen können;

- schaffen Öffentlichkeit, können damit die Arbeitsprozesse und -ergebnisse für Anregungen und konstruktive Kritik von außen öffnen und zur Integration der Klienten in andere soziale Systeme beitragen;

- bilden eine eigenständige gesellschaftliche und kulturelle Praxis, an der die Klienten bzw. Lernenden durch den Medieneinsatz aktiv handelnd teilhaben können, eine Praxis, die eigene Regeln vorgibt, aber auch neue Handlungsorientierungen bietet (vgl. Merkert 1992; vgl. Boeckmann 1994).

- Durch mediale Bedingungsfaktoren im Berufsfeld wird die thematische Progression der pädagogischen Kommunikationsprozesse bestimmt, d.h. der methodische Ablauf des beruflichen Handelns und der damit angestrebten Lernprozesse bei den Klienten. Das gilt für alle Erziehungs-, So- 
zialisations-, Lern- und Bildungsprozesse, sowohl in den Bereichen der Früherziehung, der Schule, der Berufsausbildung und Fortbildung, der Erwachsenen- und Altenbildung, als auch für sozialpädagogische Arbeitsfelder, z.B. in der Heimerziehung, der Kinder- und Jugendarbeit, der interkulturellen Erziehung oder der Arbeit in sonderpädagogischen Einrichtungen und erst recht für alle medienpädagogischen Berufe, z.B. im Bereich der Entwicklung von Multimediasoftware oder Lernprogrammen.

- Da alle pädagogischen Handlungen einer medialen Vermittlung bedürfen, wird durch die Art der medialen (Re-) Präsentation auch die Anschlußrationalität bestimmt, d.h. die Art und Weise, wie Kommunikationen aneinander anschließen. Egal, auf welchen Zeichensystemen die Kommunikationsmedien beruhen, sie sind interpretierbar und müssen interpretiert werden. Je konkreter und bildhafter, je vielfältiger die Zeichenkombinationen (z.B. Videofilm oder Multimedia), desto größer die Interpretationsmöglichkeiten und die geforderten Interpretationsleistungen. Desto größer ist aber auch die Chance für alle an einem pädagogischen Kommunikationsprozeß Beteiligten, an diesen Prozeß mit eigenen Vorstellungen und Ideen, Erfahrungen und Argumenten, Wünschen und Befürchtungen anschließen zu können. Damit erhöht sich die Chance für eine Verständigung, für subjektive Lernprozesse, für die gemeinsame Entwicklung von Problemlösungen und damit für die Erreichung der pädagogisch angestrebten Ziele.

- Weitere Veränderungen der Sachstruktur pädagogischer Kommunikationen können sich infolge der Digitalisierung und der Verbindung unterschiedlicher Medien auf der gemeinsamen Plattform des Multimediacomputers ergeben:

- Integration: Mit Hilfe eines Gerätes (Computers) können auf der Grundlage digitalisierter Daten unterschiedliche Zeichensysteme, Bilder, Sprache, Töne, Graphiken, Bewegtbilder usw. gemeinsam und in vielfältigen Kombinationen dargestellt werden. Wichtig ist dabei, daß die gespeicherten Informationen im Gegensatz zu linearen sprachlichen Texten eine sog. Hypertext-Struktur aufweisen, d.h. durch „links“ verbundene, nicht-lineare „Text“-Bausteine beinhalten.

- Interaktivität: Der Computer als Hypermedium erlaubt somit (je nach Software) eine beliebige, selbstgesteuerte Auseinandersetzung mit diesen Inhalten, ermöglicht die Speicherung und Weiterverarbeitung von Eingaben und gibt Rückmeldung über die aus geführten Routinen.

- Vernetzung: Durch den lokalen, regionalen oder weltweiten Zusammenschluß von Computern (Intranet oder Internet) ergeben sich völlig neue Nutzungsmöglichkeiten (Informationsbeschaffung im WWW oder Informationsaustausch über Email). 
3.2 Veränderungen des professionellen pädagogischen Handelns aufgrund der Veränderungen der Sozialstruktur und der Zeitstruktur

Die Entwicklungen im Medienbereich haben neue Möglichkeiten der Information und Kommunikation, der Unterhaltung und Entspannung, der Teilhabe an der Politik und Kultur, der Bearbeitung und Lösung von Aufgaben geschaffen, aber ebenso neue Herausforderungen und Probleme, neue Lernzwänge, Orientierungsverluste und Konflikte mit sich gebracht. Dadurch hat sich die Komplexität sozialer Systeme erheblich vergrößert. Das trifft auch auf die Eigenkomplexität pädagogischer Arbeitsfelder zu. Durch den vielfältigen und flexiblen Einsatz von Medien können neue, distanzierte Kontaktformen und soziale Strukturen mit bisher nicht gekannten Lernmöglichkeiten entstehen, wenn z.B. Lernende untereinander oder mit den Pädagogen über Email kommunizieren. Die Klienten können dabei lernen, ihre Lern- und Problemlösungsprozesse in größerem Umfang selbständig zu organisieren. Die selbstverständliche Nutzung der Medien zur Problemlösung und Aufgabenbewältigung fördert gleichzeitig ihre Medienkompetenz.

Von besonderer Bedeutung für die gemeinsame Arbeit und für die Weiterentwicklung der individuellen Lerninstrumente ist eine kritische Reflexion der Lernwege und der medial dokumentierten Arbeitsergebnisse in einer Gruppe. Die Präsentation von gemeinsamen Arbeits- bzw. Projektergebnissen ist eine hervorragende Gelegenheit zur wiederholten und vertieften Auseinandersetzung mit dem Thema und zu einer schärferen sprachlichen, d.h. abstrakt-begrifflichen Fassung der wesentlichen Erkenntnisse. Diese personale sprachliche Kommunikation trägt zur Ausbildung eines gemeinsamen Wissens bei, das ein zentrales Fundament jedes sozialen Zusammenlebens in der Gesellschaft darstellt. Pädagogische Handlungssysteme müssen sich daher als lernende soziale Systeme verstehen, in denen der einzelne Lernende nur soviel für sich und die Lösung seiner Probleme profitieren kann, wie sich das gesamte Handlungssystem als lernendes System weiterentwickelt. Damit ist also die Kreativität und Phantasie der Pädagogen gefordert, damit in vielfältigen sozialen Formen die Aktivierung und der selbstgesteuerte und flexible Einsatz der individuell verfügbaren Lerninstrumente zu einer vertieften Auseinandersetzung mit Inhalten, Themen und Problemen und zur Weiterentwicklung ihres Lernapparats führt.

Veränderungen des Zeitparameters durch Medieneinsatz in den pädagogischen Handlungssystemen lassen sich nur schwer abstrakt beschreiben. Aber es ist unmittelbar einsichtig, daß durch die medialen Möglichkeiten zur Informationsbeschaffung, Kontaktaufnahme, Datenspeicherung und Dokumentation von Arbeitsprozessen und -ergebnissen die zeitlichen Strukturen professioneller pädagogischer Handlungsprozesse erheblich verändert werden können. 


\section{Welches Wissen und Können müßte Bestandteil einer medienpädagogischen Grundqualifikation sein?}

Die bisherigen Überlegungen zeigen, daß eine medienpädagogische Grundqualifikation praktisch in allen Dimensionen pädagogischer Professionalität (nach K.O. Bauer 1998, S. 346) zu verankern ist.

1) Sie muß integraler Bestandteil des beruflichen Selbst und der berufstypischen Werte und Ziele als Kern dieses Selbst sein. D.h. jeder Berufspädagoge kommt nicht umhin, sich in einem gewissen Sinn als medienpädagogischer Fachmann zu verstehen. Sein Selbst als organisierendes Handlungszentrum muß für die medialen Aspekte in jeder Handlungssituation sensibel sein und mediale Einflüsse hinsichtlich ihrer pädagogischen Ambivalenz in der Verbindung von Wahrnehmung, Wissen und Handlungsrepertoire angemessen berücksichtigen können.

2) Professionalität bedeutet, daß Pädagogen ihre Handlungen unter Bezug auf eine Berufswissenschaft begründen und sich in einer nicht alltäglichen Berufssprache verständigen. D.h. die Erziehungswissenschaft als zuständige Fachdisziplin muß heute die Medienpädagogik in einem umfassenden Verständnis als wesentliches Teilgebiet einschließen.

Unter den beschriebenen Bedingungen brauchen alle Pädagogen nicht nur ein solides Wissen über die Medien (alle Medien!) und deren technische Möglichkeiten, über Medienentwicklungen, über pädagogisch geeignete Software und deren Möglichkeiten und Grenzen, über fachspezifische Informationsquellen im Internet. Damit ist umschrieben, was heute neben der Medienkompetenz als informationstechnische Bildung umschrieben wird. Für ihre Handlungsplanung müssen die Pädagogen auch wissen, auf welches spezifische Zeichenrepertoire die einzelnen Medien und insbesondere Multimedia aufbauen. Sie brauchen ein zeichen- und kommunikationstheoretisches Grundwissen, um diese Zeichensysteme zur Erreichung ihrer pädagogischen Ziele adäquat einsetzen zu können. Sie müssen wissen, welche visuellen und auditiven, symbolischen und ikonischen, analogen und digitalen, registrativen und kreativ-imitativen Elemente die einzelnen Medien in ihrer Zeichenwelt vereinen, welche Wahrnehmungserlebnisse sie erlauben und wie sie die vieldeutige Welt der Anzeichen in pädagogische Kommunikationssituationen hereinholen können. Sie sollten in der Lage sein, dieses Wissen zur Gestaltung pädagogischer Handlungsfelder kreativ einzusetzen, um gemeinsam mit den Klienten die gewünschten Lernhilfen erarbeiten zu können.

Zur Begründung ihres Handelns benötigen Professionelle Pädagogen auch Kenntnisse aus der medienpädagogischen Forschung, über Medienwirkungen und über die Mediennutzung in der Freizeit, über Medienpräferenzen und die Einstellungen zu den Medien, über das angeeignete Medienwissen und die verfügbaren Medienkompetenzen der Klienten. Ohne ein solches Wissen werden die Pädagogen die Lernenden und Hilfebedürftigen in ihren Problemen und Zielen, in ihrem Denken, Fühlen und Handeln oft kaum mehr 
verstehen können! Um überhaupt angemessene Lernhilfen geben zu können, müssen sie in der Lage sein, an die Medienwelten der Klienten anzuknüpfen und ihre Medienerfahrungen zu nutzen.

3) Im Zentrum pädagogischer Professionalität steht ein berufsspezifisches Handlungsrepertoire zur Bewältigung der Arbeitsaufgaben. D.h. daß das medienpädagogische Wissen durch eine entsprechende Handlungsfähigkeit in Form von Medienkompetenz zu ergänzen ist. Das betrifft zunächst die neuen Medien, insbesondere dort, wo sie als Lehr- und Lernmittel bedeutsam sind. Für die Erziehungsarbeit mit Kindern und Jugendlichen, aber auch für alle Felder des Unterrichts und der Bildungsarbeit sind darüber hinaus Erfahrungen und spezifische Fähigkeiten in der praktischen Medienarbeit von großem Nutzen. In allen Dimensionen des beruflichen Handlungsrepertoires, die empirisch ermittelt worden sind, - das sind soziale Strukturen bilden, Interagieren, Kommunizieren, Gestalten und Hintergrundarbeit (vgl. Bauer 1998) -, eröffnen praktische Fähigkeiten im Umgang mit Medien eine Vielzahl an interessanten und flexiblen Handlungsalternativen.

4) Schließlich ist es wichtig zu betonen, daß die Übernahme von persönlicher Verantwortung für Handlungsfolgen im eigenen Einflußbereich als weiteres Merkmal von Professionalität ohne die beschriebenen medienpädagogischen Qualifikationen unter den Bedingungen einer mediengeprägten Alltagswelt kaum noch möglich erscheint.

\section{Konsequenzen für die Ausbildung}

Aus der gewählten systemischen Betrachtungsweise ergibt sich ganz eindeutig, daß die Wissensvermittlung in den Bereichen „Medienpädagogik“ oder/ und „Informationstechnische Bildung“ im Studium oder später in der Fortbildung zwar eine notwendige, aber keineswegs hinreichende Bedingung für eine angemessene Ausbildung medienpädagogischer Kompetenz bei Pädagogen darstellt. Wenn sich in einer von Medien geprägten Alltagswelt und durch den Einsatz von Medien alle Beziehungsmuster in den pädagogischen Handlungssystemen verändern, dann müssen auch alle beruflichen Tätigkeiten, alle Erziehungs-, Unterrichts-, Lern- und Bildungsprozesse unter Berücksichtigung dieser Dimension der symbolischen Vermittlung und der daraus folgenden methodischen Konsequenzen neu gesehen werden. Dann wird es darauf ankommen, in allen Bereichen der Aus- und Fortbildung diese veränderte Perspektive sichtbar zu machen und einzuüben. Die Medienpädagogik kann im Rahmen der Erziehungswissenschaft oder als eigenes Fach zwar die dafür erforderlichen Erkenntnisse, Theorien und praktischen Konzepte bereitstellen. Aber diese veränderte Sichtweise auf die Erziehungs-, Ent- wicklungs-, Lern- und Bildungsprozesse unter besonderer Berücksichtigung ihrer medialen Dimension muß auch in anderen Disziplinen (z.B. in der Psy- 
chologie, Soziologie, in der Lehrerausbildung in den Fachdidaktiken) vermittelt und in ihren Konsequenzen für das berufliche Handeln deutlich gemacht werden. Daraus ergibt sich, daß die erforderlichen Kenntnisse und Fähigkeiten nur in einer integrierten medienpädagogischen Ausbildung sinnvoll und effektiv vermittelt werden können (vgl. Spanhel 1997b).

\subsection{Aufgabenfelder und Inhalte im Bereich der Erziehungswissenschaft}

Ein erstes Aufgabenfeld betrifft die Frage nach der Veränderung des Erziehungs- und Bildungsauftrags der Schule im Zusammenhang mit den Entwicklungen im Medienbereich, die in der Erziehungswissenschaft selbst noch nicht zufriedenstellend geklärt ist. Trotzdem müssen im Lehramtsstudium folgende Fragen behandelt werden:

- Welche Erziehungsdefizite und -probleme, die in der modernen Informationsgesellschaft in Familie und Freizeit entstehen, muß die Schule überwinden, damit überhaupt Lernen und Unterricht möglich werden? Medienerziehung macht dabei nur einen Teilaspekt aus. Welche Möglichkeiten dazu bietet der Medieneinsatz?

- Wie können die Medienerfahrungen der Klienten und die dabei erworbenen Lernformen sowie Wissensinhalte in die pädagogische Arbeit integriert werden?

- Wie sind angesichts der Bedeutung von Methoden- und Medienkompetenz der Bildungs- und der Lernbegriff neu zu fassen?

Ein zweites Aufgabenfeld betrifft die Frage nach der Veränderung der pädagogischen Berufsrolle im Zusammenhang mit dem verstärkten Einsatz der neuen Medien in den pädagogischen Arbeitsfeldern: In der Erziehungswissenschaft müssen sich die angehenden Pädagogen ausreichende Kenntnisse über Grundfragen und -begriffe, Forschungsergebnisse, Theorien und praktische Konzepte der Medienpädagogik aneignen, damit sie später den Klienten die erforderliche Medienkompetenz vermitteln können.

Als Ausgangspunkt für eine Fachintegration der Medienpädagogik in die Erziehungswissenschaft wäre zu fragen:

- Wie verändern die Medienwelten der Klienten ihre Lern-, Sozialisations und Entwicklungsprozesse und die Prozesse der sozial-moralischen Erziehung während des gesamten Lebenslaufs?

- Wie wandeln sich pädagogische Handlungssysteme in einer mediengeprägten Alltagswelt und wie verändern sich dadurch die Pädagogenrolle und das pädagogische Verhältnis? (vgl. Merkert 1992).

Die Beschäftigung mit diesen theoretischen Fragestellungen sollte die Studierenden befähigen und anregen, sich um genaue Einblicke und ein vertieftes Verständnis der Medienwelten ihrer Schüler, ihrer Sichtweise der Medien, ih- 
re Medienpräferenzen und um die mit ihrem Medienhandeln verbundenen Sinnorientierungen zu bemühen.

Ein weiteres wichtiges Aufgabenfeld liegt im Bereich der Methodenkompetenz. Die erleichterte Handhabung, die Verfügbarkeit an jedem Ort und zu jeder Zeit und die große Anpassungsfähigkeit durch Software macht heute die Medien zu vielfältig und flexibel einsetzbaren Werkzeugen zur Aufgaben- und Problemlösung, zu Instrumenten des Lehrens und Lernens, zu Mitteln der Kommunikation zwischen den Mitgliedern einer Gruppe und nach außen, zu Mitteln des persönlichen Ausdrucks, der Gestaltung, der Dokumentation, z.B. von Gruppenergebnissen, Projekten oder Aktivitäten und zu Instrumenten der Kontaktherstellung zu anderen Lernenden oder Lerngruppen. Das bedeutet, daß im Zusammenhang mit dem Medieneinsatz die traditionellen Methoden des professionellen Handelns, seien es Erziehungs- oder Lehr-Lernmethoden, sozialpädagogische oder Interventionsmethoden neu durchdacht und an diese medialen Bedingungen angepaßt werden.

\subsection{Aufgabenfelder und Inhalte in der praktischen Ausbildung}

Durch die Einrichtung von Medienwerkstätten an den Universitäten müßte sichergestellt werden, daß schon während des Studiums der Umgang mit den neuen Medien (Computer, Multimedia, Internet) praktisch eingeübt werden kann. Aber auch in der Arbeit mit den traditionellen technischen Medien (Fernsehen, Videofilme, Hörmedien) sollten die Studierenden eine größere Vertrautheit und Sicherheit gewinnen und grundlegende Fertigkeiten und Arbeitstechniken (der Medienanalyse wie der Produktion) intensiver einüben können. In den Praktika wäre darauf zu achten, daß sie die Anwendung dieser medienspezifischen Arbeitstechniken in variablen pädagogischen Kontexten erproben und reflektieren können. Sie müssen dabei einen Überblick über vorhandene Software erhalten und die Zugänge zu medienpädagogischen Materialien kennenlernen. Im Rahmen konkreter Fallanalysen sollten die Studierenden befähigt werden, die Einflüsse der Medienwelten auf pädagogische Handlungssituationen, auf die Klienten und ihre Lernprobleme und auf die Pädagogen selbst sensibel wahrzunehmen und in ihren Wirkungen systematisch zu analysieren.

\section{Literatur}

Aufenanger, St.: Die neuen Medien und die Pädagogik. In: Bildung und Erziehung, 49 (1996) 4, S. 449-460

Aufenanger, St.: Verantwortung und Gerechtigkeit in bezug auf Neue Medien. Gedanken und Vorschläge für die Umsetzung im schulischen Unterricht. In: Schmälzle, U.F. (Hrsg.): Neue Medien - Mehr Verantwortung. Bundeszentrale für politische Bildung. Bonn 1992, S. 99-122

Bauer, K.-O.: Pädagogisches Handlungsrepertoire und professionelles Selbst von Lehrerinnen und Lehrern. In: Zeitschrift für Pädagogik, 44 (1998) 3, S. 343-354 
Boeckmam, K.: Unser Weltbild aus Zeichen. Zur Theorie der Kommunikationsmedien. Wien 1994

Forum Info 2000 (Hrsg.): Bildung und Medienkompetenz im Informationszeitalter. Arbeitsgruppenbericht AG 4. Bonn 1998

Giesecke, H.: Pädagogik als Beruf. Grundformen pädagogischen Handelns. Weinheim 1996

Hermanns, A.: Erziehungswissenschaft. Einführung in die Grundstruktur des Fachs und Erschließung fachspezifischer Texte. Uni-Training. Stuttgart 1995

Krüger, H-H./Helsper, W. (Hrsg.): Einführung in Grundbegriffe und Grundfragen der Erziehungswissenschaft. Opladen 1995

Lassalm, R.: Grundriß einer Allgemeinen Pädagogik. Heidelberg 1977

Luhmam, N.: Soziale Systeme. Frankfurt/M. 1985

Merkert, R.: Medien und Erziehung. Darmstadt 1992

Moser, H.: Einführung in die Medienpädagogik. Opladen 1999

v. Renn, (Hrsg.): Medienkompetenzen als Schlüsselbegriff. Bad Heilbronn 1996

Spanhel, D.: Das Lernen optimieren. Neue Chancen durch den Einsatz von Informationsund Kommunikationstechniken. In: Medienimpulse, 3 (1994) 9, S. 64-73

Spanhel, D.: Die pädagogische Problematik der Medien. Konsequenzen für die schulische Medienerziehung. In: Miedaner, M. (Hrsg.): Familienmitglied Fernseher? Neuried 1995, S. 134-160

Spanhel, D. (a): Erziehung in einer mediengeprägten Alltagswelt. Probleme und Handlungsmöglichkeiten. In: Liedtke, M. (Hrsg.): Kind und Medien. Zur kulturgeschichtlichen und ontogenetischen Entwicklung einer Beziehung. Bad Heilbrunn 1997, S. 229-247

Spanhel, D. (b): Anforderungen an die Lehrerausbildung auf der Basis von Erfahrungen in Modellversuchen zur Medienerziehung. Unveröff. Vortragsmanuskript. Nürnberg 1997

Treml, A.K.: Lernen. In: Krüger, H.-H./Helsper, W. (Hrsg.): Einführung in Grundbegriffe und Grundfragen der Erziehungswissenschaft. Opladen 1995, S. 93-102

Tulodziecki, G.: Handlungsorientierte Medienerziehung in Beispielen. Bad Heilbrunn 1995 Tulodziecki, G./Blömeke, S. (Hrsg.): Neue Medien - neue Aufgaben für die Lehrerausbildung. Tagungsdokumentation. Gütersloh 1997 